\title{
Dispersion Management in Optical Soliton Transmission Systems: A Review
}

\author{
Harsimran Singh ${ }^{1}$, Manjit Singh ${ }^{2}$ \\ Guru Nanak Dev University Regional Campus, Jalandhar, Punjab ${ }^{1,2}$
}

\begin{abstract}
We have reviewed various paper such as "Stabilization of dispersion-managed solitons in random optical fibers by strong dispersion management" and "Simulative analysis of dispersion managed solitons for Long-haul optical communication system". They have investigated the stability of DM solitons that is based on a technique of separation of scales. It provides a fast and efficient way to compute all relevant quantities. They have given precise asymptotic expressions for the widths and chirps of the stable solitons, as well as conditions for their stabilities in homogeneous and inhomogeneous media. The solitons corresponding to strong dispersion managements are more stable with respect to the influence of random fluctuations of the chromatic dispersion than the solitons corresponding to weak dispersion managements. The other paper gives the design, implementation and performance analysis of a dispersion managed soliton link. The soliton transmission is very attractive as a potential method for realizing highspeed, long distance communication. The performance analysis includes 3D diagram, dispersion mapping and optical spectrum analysis. Analysis shows that by using dispersion compensated fiber and periodic amplification with EDFA, soliton based data transmission with least error is possible.
\end{abstract}

Keyword: Solitons, Dispersion Compensation, Amplifiers, Dispersion-managed Soliton, Random Dispersion.

\section{INTRODUCTION}

Dispersion management is a somewhat wider term than dispersion compensation, even though both are often used in the same way. Strictly, dispersion compensation should be understood as a method for canceling the chromatic dispersion of some optical element(s), whereas dispersion management is more generally the use of tailored dispersion properties in order to enhance some function.

Optical solitons are spatially localized, pulse-like, nonlinear waves that almost retain their shapes while propagating in ideal lossless fibers. This steady propagation stems from an exact balance between nonlinear and dispersion terms in the conservative form of the nonlinear Schroedinger equation which describes ideal fibers. The fact that solitons are spatially localized and propagate with (little to) no deformation makes them the carriers of choice for modern optical communication, as they can be used to encode a bit in a small amount of space through the presence or absence of the pulse in a designated temporal window. However, the balance between nonlinearity and dispersion is not exact in real optical fibers, which may cause the pulse to broaden and "spill over" the designated window. This can result in transmission errors or, if the window size is increased to avoid spill-over, in a reduction of the transmission rate.

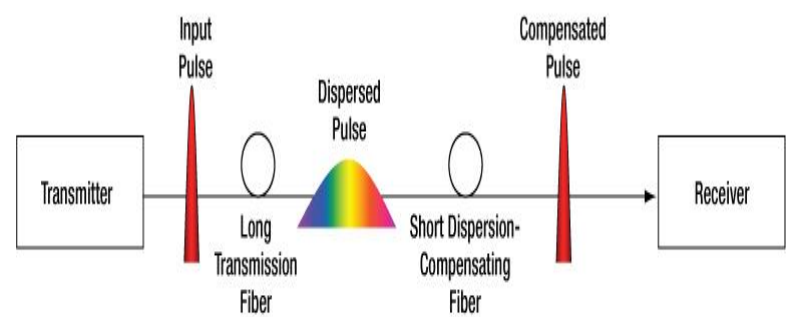

Fig. 1: Dispersion Compensating Fiber

While error-correcting codes can be used at the receiving end to compensate for dispersion-induced errors, all-optical approaches are typically preferred because they do not affect the speed at which information is processed. A typical alloptical dispersion-management technique consists of periodically alternating lengths of fibers with positive and negative group-velocity dispersions, so as to compensate for pulse broadening on average. While traveling through the fiber, the pulse experiences broadening and recompression so that, at the end of each compensation element, the width and frequency chirp of the pulse are restored to the initial desired values. This method is effective if both fiber nonlinearity and residual dispersion only slightly affect the evolution of the pulse over one compensation period. If this is not the case, these dispersion maps may fail to work appropriately, especially if the characteristics of the fiber are uncertain. 
From a control theory perspective, this (passive) dispersion technique can be seen as open-loop control, where the group-velocity dispersion is the input, pulse width is the output, and the goal is to regulate the output to its desired value at a fixed propagation length. With this analogy in mind, it seems tempting to try and use feedback control to improve dispersion management of optical solitons and, in particular, address the problem of soliton transmission along uncertain fibers, provided dispersion can be precisely tuned and pulse deformation can be precisely measured in realtime along the fiber. In this sense, several new technologies have been developed recently that allow for some degree of continuous dispersion tuning, from microfluidics-based tunable dispersion materials to fiber-based approaches ranging from fiber gratings to higher-order mode fibers, to name but a few.

While a number of challenges still need to be overcome to arrive at spatially continuous, fine resolution, sensing and actuation, the time seems ripe for the investigation of advanced control techniques for active dispersion management. Pioneering work on this area was done by Koehn and Langbort in 2010, where a nonlinear state-feedback control law was derived based on controlled Hopf bifurcation. However, similar to the existing passive techniques, this approach requires a priori precise knowledge of the characteristics of the fiber in order to achieve dispersion correction. To overcome this limitation, we are developing an L1 adaptive control scheme for the problem of active dispersion management for propagation of solitons along uncertain fibers.

\section{Problems in Conventional Soliton Transmission}

In lossless fibers with constant GVD, isolated solitons can propagate over unlimited distances without distortion. Actual fibers have small but finite losses requiring amplification for long-distance unregenerated transmission. Laser amplifiers such as erbium-doped fiber amplifiers are usually used, where amplified spontaneous-emission (ASE) noise is inevitably added to the signal at each amplification. The noise modulates soliton frequency randomly, which leads to random timing jitter through GVD of the fiber. The timing jitter is called the Gordon-Haus timing jitter and was introduced in Sect. 6.5. The magnitude of the Gordon-Haus timing jitter (variance of the fluctuation of arriving time of a pulse) is proportional to the GVD of the fiber when pulse width is kept constant. We can expect smaller Gordon-Haus timing jitter when we reduce the fiber GVD. The GVD, however, cannot be decreased arbitrarily because the soliton energy, and consequently the signal-to-noise ratio (SNR) at the receiver, is proportional to the fiber GVD. Thus the Gordon-Haus timing jitter sets a fundamental limit to soliton transmission.

The translation from frequency fluctuations to timing jitter through fiber GVD is a major mechanism of performance degradation of soliton systems. Not only the Gordon - Haus effect but also the interaction between adjacent pulses and the interaction between soliton and acoustic waves are reduced for small fiber GVD, but with a limit placed by the SNR requirement.

The nonlinear interaction between adjacent pulses through exponential tails of sech pulses inhibits packing solitons with small separation. Separations larger than about five times the pulse width are usually needed. This means that the spectral width of the signal is considerably larger than that of modulation formats used in linear transmission, such as an NRZ format,

\section{LITERATURE REVIEW}

N.J. Smith et.al in [1] examined the formation of stable soliton-like pulses in optical fibres with a periodic dispersion map. It is found that increased energy is required to launch a pulse of given width compared to the equivalent uniform fibre with equal path-average dispersion. The stable pulse shapes in dispersion managed systems are significantly different to the hyperbolic secant solitons found in uniform fibres.

J.H.B. Nijhof et.al in [2] discovered numerical modelling that there are stable nonlinear transmissions pulses for periodically dispersion managed systems where the path average dispersion may be either anomalous, zero, or even normal.

Akira Hasegawa et.al in [3] reviewed recent progress in fiber dispersion management technologies to achieve enhancement in transmission rates of the soliton-based wavelength division multiplexing (WDM) as well as time division multiplexing. This review covers the basic concept of dispersion management, its effectiveness in WDM and in elongation of amplifier spacing, optimization in stepwise dispersion profiles, and generation of solitons with more desirable properties (i.e., dressed solitons and quasi-solitons).

Sergei K. Turitsyn et.al in [4] examined the properties of dispersion-managed (DM) solitons in fiber lines with dispersion-compensation period $L$ much shorter than amplification distance $Z a$. DM solitons in systems with shortscale dispersion compensation have reduced power compared with DM solitons in previously studied regimes with $L \geq Z a$ power (for the same local and average dispersion and the same pulse width). As a result, chirped DM soliton transmission with reasonably low power can be realized with shorter pulses when DM solitons in systems with large $L / Z a$ ratios have energies that are too high to be used in practice.

Sergei K. Turitsyn et.al in [5] analyzed different schemes of the dispersion management using a variational approach developed in their previous works. They demonstrate how to exploit this variational method to determine in a simple 
way the energy enhancement of the dispersion-managed soliton, and the optimal point for launching of the signal, as well as how to evaluate characteristics of a carrier signal for specific system parameters. The variational method is a useful and effective tool for design and optimization of dispersion-managed communication systems.

Amitabha Banerjee et.al in [6] presented a comprehensive review of various aspects of WDM-PONs proposed in the literature. This includes enabling device technologies for WDM-PONs and network architectures, as well as the corresponding protocols and services that may be deployed on a WDM-PON. The WDM-PON will become a revolutionary and scalable broadband access technology that will provide high bandwidth to end users.

N.J. Frigo et.al in [7] demonstrated a passive optical network (PON), based on a wavelength-division multiplexing router, with modulators (instead of lasers) at the subscriber terminals. A single, cost-shared, tunable laser is timedivision multiplexed to provide bidirectional switched WDM services for $\mathrm{N}$ subscribers with independent formats and bit-rates for each. Simultaneous support of telephony and compressed digital video are presented in which RF subcarrier multiplexing is used to provide service segregation and resolve upstream packet contention.

Soo-Jin Park et.al in [8] proposed and implemented a WDM-PON system as a platform for TPS. The system employs an amplified spontaneous emission (ASE)-injected Fabry-Pérot laser diode scheme. It has 32 channels of $125 \mathrm{Mb} / \mathrm{s}$ and adopts Ethernet as Layer 2. Multicast and virtual local area network features are used for the integration of services such as Internet protocol high-definition broadcast, voice-over Internet protocol, video on demand, and video telephone. The services were demonstrated using the WDM-PON system.

Yuanqiu Luo et.al in [9] summarized the TWDM-PON research in FSAN by reviewing the basics of TWDM-PON and presenting the world's first full-system $40 \mathrm{~Gb} / \mathrm{s}$ TWDM-PON prototype. After introducing the TWDM-PON architecture, they explore TWDM-PON wavelength plan options to meet the NG-PON2 requirements. TWDM-PON key technologies and their respective level of development are further discussed to investigate its feasibility and availability. The first full-system $40 \mathrm{~Gb} / \mathrm{s}$ TWDM-PON prototype is demonstrated to provide $40 \mathrm{~Gb} / \mathrm{s}$ downstream and $10 \mathrm{~Gb} / \mathrm{s}$ upstream bandwidth. This full prototype system offers $38 \mathrm{~dB}$ power budget and supports $20 \mathrm{~km}$ distance with a 1:512 split ratio. It coexists with commercially deployed Gigabit PON (G-PON) and 10 Gigabit PON (XG-PON) systems. The operator-vendor joint test results testify that TWDM-PON is achievable by the reuse and integration of commercial devices and components.

Chang-Joon Chae et.al in [10] proposed and demonstrated a novel implementation of optical carrier-sense multiple access with collision detection to efficiently transport Ethernet frames over a star coupler (SC) based passive optical network. They introduce a redirection mechanism at the SC to achieve high channel efficiency.

\section{STABILIZATION OF DISPERSION-MANAGED SOLITONS IN RANDOM OPTICAL FIBERS BY} STRONG DISPERSION MANAGEMENT

The propagation of dispersion-managed solitons in optical fibers with randomly perturbed dispersion maps is considered. The interplay between the periodic dispersion management and the random dispersive fluctuations is precisely analyzed. Analytic expressions are derived for the moments of the pulse widths as well as for the probability density functions. It is shown that a strong dispersion management stabilizes the soliton, while a small anomalous residual dispersion is necessary for preventing from a stochastic resonance phenomenon. Analytical results are confirmed by direct numerical simulations.

\section{SIMULATIVE ANALYSIS OF DISPERSION MANAGED SOLITONS FOR LONG-HAUL OPTICAL COMMUNICATION SYSTEM}

They proposed to demonstrate the dispersion-managed soliton regime in a fiber link with the loss and periodical amplification upto $36000 \mathrm{Km}$. Ideal lossless and loss-managed solitons require GVD parameter $\beta 2$ to stay constant along the fiber length. Modern WDM light wave systems employ dispersion management to compensate for cumulative dispersion and to suppress FWM penalties. It was demonstrated that solitons can even form when $\beta 2$ varies along the fiber length but their properties are quite different.

\section{CONCLUSION}

In this review paper we have studied and mentioned the various aspects of Dispersion Management using Optical Solitons. We have reviewed various paper such as "Stabilization of dispersion-managed solitons in random optical fibers by strong dispersion management" and "Simulative analysis of dispersion managed solitons for Long-haul optical communication system". They have investigated the stability of DM solitons that is based on a technique of separation of scales. It provides a fast and efficient way to compute all relevant quantities. They have given precise asymptotic expressions for the widths and chirps of the stable solitons, as well as conditions for their stabilities in homogeneous and inhomogeneous media. The solitons corresponding to strong dispersion managements are more 
stable with respect to the influence of random fluctuations of the chromatic dispersion than the solitons corresponding to weak dispersion managements. The other paper gives the design, implementation and performance analysis of a dispersion managed soliton link. The soliton transmission is very attractive as a potential method for realizing highspeed, long distance communication. The performance analysis includes 3D diagram, dispersion mapping and optical spectrum analysis. Analysis shows that by using dispersion compensated fiber and periodic amplification with EDFA, soliton based data transmission with least error is possible.

\section{REFERENCES}

[1] Smith, N.J., Knox, F.M., Doran, N.J., Blow, K.J. and Bennion, I., 1996. Enhanced power solitons in optical fibres with periodic dispersion management. Electronics Letters, 32(1), pp.54-55.

[2] Nijhof, J.H.B., Doran, N.J., Forysiak, W. and Knox, F.M., 1997. Stable soliton-like propagation in dispersion managed systems with net anomalous, zero and normal dispersion. Electronics Letters, 33(20), pp.1726-1727.

[3] Hasegawa, A., Kodama, Y. and Maruta, A., 1997. Recent progress in dispersion-managed soliton transmission technologies. Optical Fiber Technology, 3(3), pp.197-213.

[4] Turitsyn, S.K., Fedoruk, M.P. and Gornakova, A., 1999. Reduced-power optical solitons in fiber lines with short-scale dispersion management. Optics letters, 24(13), pp.869-871.

[5] Turitsyn, S.K. and Shapiro, E.G., 1998. Variational approach to the design of optical communication systems with dispersion management. Optical Fiber Technology, 4(2), pp.151-188

[6] Banerjee, A., Park, Y., Clarke, F., Song, H., Yang, S., Kramer, G., Kim, K. and Mukherjee, B., 2005. Wavelength-division-multiplexed passive optical network (WDM-PON) technologies for broadband access: a review [Invited]. Journal of optical networking, 4(11), pp.737-758.

[7] Frigo, N.J., Iannone, P.P., Magill, P.D., Darcie, T.E., Downs, M.M., Desai, B.N., Koren, U., Koch, T.L., Dragone, C., Presby, H.M. and Bodeep, G.E., 1994. A wavelength-division multiplexed passive optical network with cost-shared components. IEEE Photonics technology letters, 6(11), pp.1365-1367.

[8] Park, S.J., Lee, C.H., Jeong, K.T., Park, H.J., Ahn, J.G. and Song, K.H., 2004. Fiber-to-the-home services based on wavelength-divisionmultiplexing passive optical network. Journal of Lightwave Technology, 22(11), p.2582.

[9] Luo, Y., Zhou, X., Effenberger, F., Yan, X., Peng, G., Qian, Y. and Ma, Y., 2013. Time-and wavelength-division multiplexed passive optical network (TWDM-PON) for next-generation PON stage 2 (NG-PON2). Journal of Lightwave Technology, 31(4), pp.587-593.

[10] Chae, C.J., Wong, E. and Tucker, R.S., 2002. Optical CSMA/CD media access scheme for Ethernet over passive optical network. IEEE Photonics Technology Letters, 14(5), pp.711-713. 